\title{
Angular position of nodes in the superconducting gap of YBCO
}

\author{
H. Aubin, K. Behnia, M. Ribault \\ Laboratoire de Physique des Solides (associé au CNRS), \\ Université Paris-Sud, 91405 Orsay, France \\ R. Gagnon and L. Taillefer \\ Department of Physics, 3600 University Street, McGill University, Montréal, Québec, Canada \\ H3A2T8
}

(August 11, 2018)

\begin{abstract}
The thermal conductivity of a $\mathrm{YBa}_{2} \mathrm{Cu}_{3} \mathrm{O}_{6.9}$ detwinned single crystal has been studied as a function of the relative orientation of the crystal axes and a magnetic field rotating in the $\mathrm{Cu}-\mathrm{O}$ planes. Measurements were carried out at several different temperatures below $\mathrm{T}_{c}(0.5 \mathrm{~K}<\mathrm{T}<25 \mathrm{~K})$ and at a fixed field of $30 \mathrm{kOe}$. A four-fold symmetry characteristic of a superconducting gap with nodes at odd multiples of 45 degrees in k-space was resolved. Experiments were performed to exclude a possible macroscopic origin for such a four-fold symmetry such as sample shape or anisotropic pinning. Our results impose an upper limit of $10 \%$ on the weight of the s-wave component of the essentially d-wave superconducting order parameter of YBCO.
\end{abstract}

74.25.Fy, 74.72.Bk, 72.15.Eb

Typeset using REVTEX 
Over the last years, there has been accumulating evidence that the pairing state of high temperature superconductors may be the so-called $d_{x^{2}-y^{2}}$ state. Among the wealth of experimental data, one can mention the results from magnetic penetration depth [1] [3], angular resolved photoemission [4], and nuclear spin relaxation rate [5], which provide strong evidence for nodes in the superconducting gap of the cuprates. Furthermore, quantum phase interference experiments [6] which look directly at the symmetry of the order parameter as a function of its argument, generally confirm the presence of the nodes and the sign change of the gap function over the Fermi surface.

Most of these experiments suggest, as a recent review by Annett et al. [7] concludes, that a $d_{x^{2}-y^{2}}$ order parameter is the most plausible candidate to describe the superconducting state in these systems. However, in the case of optimally-dopped $\mathrm{YBa}_{2} \mathrm{Cu}_{3} \mathrm{O}_{7-\delta}(\mathrm{YBCO})$, it has been argued that a possible slight s-wave component could arise from the orthorhombic distortion [8]. The in-plane anisotropy observed below $\mathrm{T}_{c}$ in thermal conductivity [9] and penetration depth [2,3] suggest that the $\mathrm{CuO}$ chains play a role in the thermodynamic and transport properties of the superconducting state. Furthermore, it was recently pointed out that the in-plane penetration depth anisotropy is difficult to understand within a proximity model with only a pure $d$-wave pairing in the $\mathrm{Cu}-\mathrm{O}$ plane $[10$. One consequence of the existence of a s-wave component would be a shift of the nodal positions from the principal diagonal directions over the Fermi surface. Thus, the precise angular location of the gap nodes on the Fermi surface would be a useful information in the current debate on the symmetry of the order parameter in YBCO.

We present here a study of the thermal conductivity of a YBCO single-crystal, $\kappa$, rotating in a magnetic field parallel to the $\mathrm{Cu}-\mathrm{O}$ planes. A pioneer work by Salamon and co-workers [11,12 showed that thanks to the Andreev scattering of quasiparticles by rotating vortices, such an experiment can be a relevant probe of the k-space anisotropy of the superconducting gap. This work was criticized by Klemm et al. [13] who contested a microscopic interpretation of the data. Moreover, error bars on the angular dependence of the longitudinal thermal conductivity were comparable to the magnitude of the periodical 
oscillations [11], making it difficult, if not impossible, to resolve a four-fold symmetry of $\kappa(\theta)$ which is the qualitative distinction of d-wave superconductivity. In this letter, we begin by reporting on high-resolution experimental results which establish such a four-fold symmetry and then we address objections to a microscopic interpretation of this angular dependence. Finally, we show that our data impose an upper limit on the relative weight of the s-wave component of the essentially d-wave state of YBCO.

Our YBCO single crystal was prepared by a self-flux method described elsewhere [14]. Measurements were performed in a dilution refrigerator using a one heater-two-thermometer steady state method. The sample was anchored at one end to a $\mathrm{Cu}$ heat sink, and a heater resistor was attached to the other. The heat current $(\mathrm{J})$ was injected along the b-axis direction (i.e. parallel to the chains), and the thermal gradient was measured along the same direction with two Lakeshore Cernox thermometers. The experimental setup was rotated at the center of a superconducting coil with the rotation axis along the c-axis and the magnetic field parallel to the $\mathrm{Cu}-\mathrm{O}$ planes. The precise relative orientation of the magnetic field and the crystal was determined through a Hall probe rigidly fixed on the setup. The maximal available rotation was about 200 degrees. Below $5 \mathrm{~K}$, due to the slightly anisotropic magnetoresistance of the thermometers, we used a dynamic method which allowed us to calibrate the thermometers for each measured $\theta$-direction.

Fig. 1 shows the thermal conductivity of the YBCO sample as a function of temperature over a wide temperature range. A few years ago, it was suggested that the steep increase of the thermal conductivity at the superconducting transition is induced by the electronic contribution in the $\mathrm{Cu}-\mathrm{O}$ planes which, due to the strongly suppressed quasiparticle scattering rate in the superconducting state, increases rapidly below $\mathrm{T}_{c}$ [9]. Early evidence for such a suppression came from microwave conductivity data [15]. Later, thermal Hall effect measurements [16] confirmed this pattern and it is now generally accepted [17] that the peak of the thermal conductivity below $\mathrm{T}_{c}$ is in large part due to electrons. In our sample this peak is higher $\left(\frac{\kappa_{\max }}{\kappa\left(T_{c}\right)}=2.2\right)$ and occurs at a lower temperature $(\sim 23 \mathrm{~K})$ than what has been previously reported [9]. Both these features point to a long maximum quasi-particle mean- 
free path in the sample studied in this work. Because of this large electronic contribution, thermal conductivity is a useful probe of the quasi-particle excitation spectrum.

The angular variation of the thermal conductivity of $\mathrm{YBCO}, \kappa(\theta)$ with $\theta=(\mathbf{b}, \mathbf{H})$, at $24 \mathrm{~K}$ is shown in fig. 2. The full range of angular variation ( $0<\theta<360$ degrees ) was obtained by inverting the direction of the magnetic field. Thermal conductivity shows a clear fourfold variation superposed on a hysteretic background. This hysteresis, observed between successive rotations, is related to the pinning of vortices, which might induce some variation of the vortex density between two consecutive crossings of the magnetic field at a $\theta$-direction. This hysteresis continues to be present at lower temperatures where the pinning is stronger. But, somewhat surprisingly, the monotonic background disappears. As shown in fig. 3a, the angular variation has a clear fourfold symmetry at $6.8 \mathrm{~K}$. This is the first time that such a symmetry of thermal conductivity is unambigously established well beyond the experimental resolution in a high- $\mathrm{T}_{c}$ superconductor.

Next, we consider possible objections to a microscopic interpretation of this fourfold symmetry such as raised by Klemm et al. [13]. These authors suggested that the angular structure might be "due to the demagnetization and flux pinning effects associated with a rectangular sample". To check this hypothesis, we performed complementary verifications. First, we used an alternative experimental procedure by rotating the crystal in the normal phase: After each rotation of about 1 degree, the sample was heated to a temperature above $\mathrm{T}_{c}(93 \mathrm{~K})$ before cooling down to the measurement temperature. The angular variation obtained in this way ( fig.2) presented the same behavior without the sloping background. Second, to rule out the possibility of a morphological origin -related to the demagnetization for example-, we studied carefully the thermal conductivity of a niobium (Nb) sample of similar shape (flat rectangular slab) and size and our results reproduce previous measurements on $\mathrm{Nb}[18]$. As shown in fig. 3b, the angular variation of the thermal conductivity for this sample has an obvious twofold symmetry. This anisotropy changes sign with decreasing temperature reflecting the nature of the dominant quasi-particle carriers [19], but the symmetry always remains twofold. Thus, the relative orientation of vortices and the applied 
current, and not the sample geometry, governs the angular structure seen in fig. 3b.

Now, with the elimination of a macroscopic origin for the fourfold variation observed in the case of YBCO, we can turn to the microscopic picture which was originally formulated by $\mathrm{Yu}$ et al. [12 and is based on the anisotropic scattering of the quasi-particles by the vortices. It is well-known [20] that the vortices have dramatic effects on the heat transport in type II superconductors. Even in the case of the cuprates with a magnetic field parallel to the basal plane, the coreless vortices lying between the $\mathrm{Cu}-\mathrm{O}$ planes can act as scatterers of the BCS-like quasiparticles. This scattering mechanism was expressed [9] in terms of Andreev reflection [21] which is a process of retroreflection of excitations where spatial variations of the amplitude or the phase of the order parameter induce branch conversion of electron-like excitations into hole-like excitations, and vice versa. In other words, the excited states distributed above the superconducting gap exhibit in the presence of a phase gradient ( superfluid flow, $\mathrm{v}_{s}$ ), a Doppler shift of their energies, $\mathrm{E}=\mathrm{E}_{0}-\mathbf{p} \cdot \mathbf{v}_{s}$, so, when a quasiparticle approaches the vortex core, its energy in the superfluid frame reaches the energy gap and is converted to a quasihole, halting its contribution to the heat transport. This scattering process is strongly dependent on the relative orientation of the quasiparticle momentum (p) and the superfluid velocity $\left(\mathbf{v}_{s}\right)$. The latter is imposed by the magnetic field. Indeed, no Andreev reflection occurs when the quasiparticle momentum is normal to the supercurrent and parallel to the magnetic field. Thus, thanks to the directionality of this scattering mechanism, thermal conductivity can identify the preferential momentum orientation of the excitations above the superconducting gap.

Qualitatively, the observed behavior, i.e. a fourfold variation with maxima for odd multiples of $45^{\circ}$, indicates that there are maxima in the angular distribution of the quasi-particle momentum in the vicinity of $\left|\mathrm{k}_{\mathrm{x}}\right|=\left|\mathrm{k}_{\mathrm{y}}\right|$. When the field is aligned along these particular directions, most quasiparticles are not Andreev reflected, and the thermal resistance is lower.

The predominance of the fourfold variation at $6.8 \mathrm{~K}$ enables us to compare the curve to a 2D version of the usual BRT expression of the electronic thermal conductivity [12]: 


$$
\kappa_{b}^{q p}=\frac{1}{2 \pi^{2} c k_{B} T^{2} \hbar^{2}} \int d^{2} p \frac{v_{g b}^{2} E_{p}^{2}}{\Gamma(\mathbf{H}, \mathbf{p})} \operatorname{sech}^{2}\left(\frac{E_{p}}{2 k_{b} T}\right)
$$

where $v_{g b}$ is the the b-axis component of the group velocity, c is the c-axis lattice parameter and the effective rate of scattering

$$
\Gamma(\mathbf{H}, \mathbf{p})=\frac{1}{\tau_{0}}\left(1+\frac{\tau_{0}}{\tau_{v_{0}}} \exp \left\{\frac{-m^{2} a_{v}^{2}\left[E_{p}-|\Delta(\mathbf{p})|\right]^{2}}{p_{F}^{2} \hbar^{2} \ln \left(a_{v} / \xi_{0}\right) \sin ^{2} \psi(\mathbf{p})}\right\}\right)
$$

depends on $a_{v}$, the intervortex mean distance, and on $\psi(\mathbf{p})$, the angle between the magnetic field and the quasi-momentum direction $\mathbf{p} . \frac{1}{\tau_{0}}$ and $\frac{1}{\tau_{v_{0}}}$ are respectively zero-field and maximum Andreev scattering rates. The gap function is a mixture of d-wave and s-wave components:

$$
\Delta=\Delta_{0}\left\{r+\frac{\cos \left(p_{x} a / \hbar\right)-\cos \left(p_{y} a / \hbar\right)}{1-\cos \left(p_{F} a / \hbar\right)}\right\}
$$

where $\mathrm{r}$ represents the relative weight of the $\mathrm{s}$ component. As mentioned above, a finite $\mathrm{r}$ would shift the nodal positions from the main diagonal directions and the angular interval between the peak positions of the thermal conductivity would differ from $90^{\circ}$. Numerical calculations using realistic physical parameters $\left(E_{F}=1 \mathrm{eV}, \tau_{0}=3 \tau_{v_{0}}=10^{-12} s\right.$ and $\left.\frac{\kappa^{q p}}{\kappa}=0.05\right)$ are shown in fig. 3a for pure $d$-wave $(r=0), d+10 \% s(r=0.1)$ and $d+30 \% s(r=0.3)$ cases. The interval $(2 \theta)$ between the two peaks is shifted from 90 degrees (pure $d-w a v e$ ) to $96(10 \%)$ and $108(30 \%)$, following the relation $2 \theta=\operatorname{Arccos}(r)$. However, in our experimental curve, the two peaks are separated by $90 \pm 6^{\circ}$. Thereafter, according to our results, the upper limit to r, i.e. the relative weight of the s component of the superconducting order parameter, $\left(\Delta=\Delta_{d}(r+\cos (2 \theta))\right)$, is about 0.1 . This is significantly lower than what is suggested through a recent examination [22] of the in-plane penetration depth anisotropy [1]. On the other hand, it is compatible with the absence of any detectable difference in the zero-field thermal conductivity along the a-axis and the b-axis at very low temperatures which also suggest an essentially d-wave state 23.

We also studied the temperature dependence of this angular variation down to $0.5 \mathrm{~K}$. Fig. 4 shows the curves for $24 \mathrm{~K}, 6.8 \mathrm{~K}$ and $0.8 \mathrm{~K}$ plotted together. A striking change in the 
structure of $\kappa(\theta)$ is detected between $6.8 \mathrm{~K}$ and $1.2 \mathrm{~K}$ where the fourfold symmetry fades away. The minimum for the field perpendicular to the thermal current (b-axis) develops significantly (i.e. $\kappa_{/ /}>\kappa_{\perp}$ ). We have found that this minimum becomes deeper with decreasing temperature or for increasing magnetic field. The origin of this crossover between these two regimes $(6.8 \mathrm{~K}$ and $1.2 \mathrm{~K})$ is not yet well understood.

However, the analysis above neglects the effect of vortices in the plane on both phonons and quasiparticles in the chains. The former may provide an explanation for the twofold variation developed at low temperatures. On the other hand, as a careful study of the inplane anisotropy of thermal conductivity at various temperatures suggests [24], the chain contribution to the thermal conductivity increases significantly below $50 \mathrm{~K}$ passing by a maximum around $15 \mathrm{~K}$. In our geometry, with the heat current applied along the chain orientation, a two-fold angular structure due to the scattering of chain quasi-particles by vortices could well be present. Additional experiments of this type on a-oriented YBCO crystals will be necessary to elucidate the effect of a rotating magnetic field on different types of heat carriers at different temperatures.

In conclusion, our results support the existence of nodes in the superconducting gap at angular positions close to what is expected for a purely d-wave state and impose an upper limit of about 0.1 to the relative weight of the s component.

We are grateful to G. Bellessa for providing us the niobium crystal. This Work was funded in part by NSERC of Canada, FCAR of Québec and the Canadian Institute for Advanced Research. L.T. acknowledges the support of the Alfred P. Sloan Foundation. 


\section{REFERENCES}

[1] Kuan Zhang et al., Phys. Rev. Lett. 73, 2484 (1994).

[2] D. A. Bonn et al., Phys. Rev. Lett. 68, 2390 (1992).

[3] W. N. Hardy et al., Phys. Rev. Lett. 70, 3999 (1993).

[4] Z.-X. Shen et al., Phys. Rev. Lett. 70, 1553 (1993); H. Ding et al., Phys. Rev. Lett. 74, 2784 (1995).

[5] J.A. Martindale et al., Phys. Rev. Lett. 68, 702 (1992).

[6] D.A. Wollman et al., Phys. Rev. Lett. 71, 2134, (1993); C. C. Tsuei et al., Phys. Rev. Lett. 73, 593, (1994).

[7] James Annett, Nigel Goldenfeld and Anthony J. Leggett http://xxx.lanl.gov/abs/condmat/9601060], to appear in Physical Properties of High Temperature Superconductors, Vol. 5, D.M. Ginsberg (ed.), (World Scientific, Singapore , 1996).

[8] See for example M.T. Béal-Monod and K. Maki, Phys. Rev. B, 53, 5775 (1996) and references therein.

[9] R. C. Yu et al., Phys. Rev. Lett. 71, 1657 (1992).

[10] W. Atkinson, J.P. Carbotte and C. O'Donovan http://xxx.lanl.gov/abs/condmat/cond-mat/9604104], To appear in the proceeding of the BICAS Summer School on "Symmetry of the Order Parameter in High-Temperature Superconductors."

[11] M.B. Salamon et al., J. of Superconductivity, 8, 449, (1995).

[12] F. Yu et al., Phys. Rev. Lett. 74, 5136 (1995); 77, 3059 (1996).

[13] R. A. Klemm et al., Phys. Rev. Lett. 77, 3056 (1996).

[14] R. Gagnon, C. Lupien and L. Taillefer, Phys. Rev. B 50, 3458 (1994).

[15] Kuan Zhang et al., Phys. Rev. Lett. 73, 2484 (1994). 
[16] K. Krishana, J.M. Harris and N.P. Ong, Phys. Rev. Lett. 75, 3529 (1995).

[17] P.J. Hirschfeld and W.O. Putikka, Phys. Rev. Lett. 77, 3909 (1996).

[18] J. Lowell and J.B. Sousa, J. Low Temp. Physics 3, 65 (1970).

[19] Kazumi Maki, Phys. Rev. 158, 397 (1967).

[20] A. Houghton and K. Maki, Phys. Rev. B 4, 843 (1971); Robert M. Cleary, Phys. Rev. 175, 587, (1968).

[21] A.F. Andreev, Sov. Phys. JETP, 19, 1228 (1964).

[22] M.T. Béal-Monod and K. Maki, to be published in Phys. Rev. B.

[23] K. Behnia et al., Synth. Metals 71, 1611 (1995).

[24] Robert Gagnon, Song Pu, Brett Ellman, and Louis Taillefer, to be published. 


\section{FIGURES}

FIG. 1. Main Panel: The temperature depencence of the thermal conductivity in a $\log / \log$ plot. Insert shows a linear plot. Note the sharp increase at $\mathrm{T}_{c}$ leading to a peak at $23 \mathrm{~K}$.

FIG. 2. Open squares and circles show the angular variation of the thermal conductivity at $\mathrm{T}=24 \mathrm{~K}$ and $\mathrm{H}=30 \mathrm{kOe}$. There are minima for the field parallel and perpendicular to the b-axis direction (heat current), and maxima when the field is parallel to the main diagonal directions. Arrows indicate the rotating direction. Solid circles indicate the results obtained with an alternative procedure of field-cooling at every angle (see text).

FIG. 3. The fourfold variation of the thermal conductivity in YBCO (a) is compared with the twofold variation for the $\mathrm{Nb}$ crystal (b). For YBCO the numerical results are shown for a pure $\mathrm{d}$-wave, $d+10 \% s-$ wave $(r=0.1)$ (solid line) and $d+30 \% s-$ wave $(r=0.3)$ (dashed line). For $\mathrm{Nb}$ the solid line is a fit using the cosine-square dependence expected for a conventional gap.

FIG. 4. Angular variation of the thermal conductivity at $24 \mathrm{~K}, 6.8 \mathrm{~K}$ and $0.8 \mathrm{~K}$, at $\mathrm{H}=30 \mathrm{kOe}$. 